First publ. in: Israel Journal of Mathematics ; 161 (2007), 1. - S. 17-27

http://dx.doi.org/10.1007/s11856-007-0070-2

\title{
A NICHTNEGATIVSTELLENSATZ FOR POLYNOMIALS IN NONCOMMUTING VARIABLES
}

\author{
IGOR KLEP AND MARKUS SCHWEIGHOFER
}

\begin{abstract}
Let $S \cup\{f\}$ be a set of symmetric polynomials in noncommuting variables. If $f$ satisfies a polynomial identity $\sum_{i} h_{i}^{*} f h_{i}=1+\sum_{i} g_{i}^{*} s_{i} g_{i}$ for some $s_{i} \in S \cup\{1\}$, then $f$ is obviously nowhere negative semidefinite on the class of tuples of non-zero operators defined by the system of inequalities $s \geq 0$ $(s \in S)$. We prove the converse under the additional assumption that the quadratic module generated by $S$ is archimedean.
\end{abstract}

\section{IntRoduction AND MAIN RESUlts}

We write $\mathbb{N}:=\{1,2, \ldots\}, \mathbb{R}, \mathbb{C}$ for the sets of natural, real and complex numbers. For $k \in\{\mathbb{R}, \mathbb{C}\}$, we consider the algebra $k\langle\bar{X}\rangle$ of polynomials in $n$ noncommuting variables $\bar{X}:=\left(X_{1}, \ldots, X_{n}\right)$ with coefficients from $k$. The elements of $k\langle\bar{X}\rangle$ are linear combinations of words in $n$ letters $\bar{X}$. The length of the longest word in such a linear combination is called the degree. We equip $k\langle\bar{X}\rangle$ with the involution * reversing the words, e.g., $\left(X_{1} X_{2}+X_{1}^{2}\right)^{*}=X_{2} X_{1}+X_{1}^{2}$. Thus $k\langle\bar{X}\rangle$ is the *algebra freely generated by $n$ symmetric elements. Let Sym $k\langle\bar{X}\rangle$ denote the set of all symmetric elements, that is, $\operatorname{Sym} k\langle\bar{X}\rangle=\left\{f \in k\langle\bar{X}\rangle \mid f=f^{*}\right\}$. A polynomial of the form $g^{*} g$ is called a hermitian square.

If $f \in k\langle\bar{X}\rangle$ is a sum of hermitian squares and we substitute bounded selfadjoint operators $A_{1}, \ldots, A_{n}$ on the same Hilbert space for the variables $\bar{X}$, then the resulting operator $f\left(A_{1}, \ldots, A_{n}\right)$ is positive semidefinite. For operators $A$ and $B$ on a Hilbert space $E$, we write $A \leq B$ (respectively $A<B$ ) to express that $B-A$ is positive semidefinite (respectively positive definite), i.e.,

$$
\begin{aligned}
& A \leq B: \Leftrightarrow\langle A v, v\rangle \leq\langle B v, v\rangle \text { for all } v \in E \\
& A<B: \Leftrightarrow\langle A v, v\rangle<\langle B v, v\rangle \text { for all } v \in E \backslash\{0\}
\end{aligned}
$$

Helton [Hel] proved (a slight variant of) the converse of the above observation: If $f \in k\langle\bar{X}\rangle$ and $f\left(A_{1}, \ldots, A_{n}\right) \geq 0$ for all self-adjoint matrices $A_{i}$ of the same size, then $f$ is a sum of hermitian squares. For a beautiful exposition, we refer the reader to $\mathrm{MP}$.

We follow the terminology and notation used (in the commutative case) by Marshall in Mar. Fix a subset $S \subseteq \operatorname{Sym} k\langle\bar{X}\rangle$. The semialgebraic 'set' (called positivity domain in [HM]) $K_{S}$ associated to $S$ is the class of tuples $A=\left(A_{1}, \ldots, A_{n}\right)$

Date: April 10, 2006.

2000 Mathematics Subject Classification. Primary 11E25, 13J30, 47L07; Secondary 08B20, 41A10, 14P10.

Key words and phrases. noncommutative polynomials, Nichtnegativstellensatz, sums of squares, semialgebraic sets, contractive operators.

Supported by the European network RAAG (EC contract HPRN-CT-2001-00271).

1

Konstanzer Online-Publikations-System (KOPS)

URL: http://nbn-resolving.de/urn:nbn:de:bsz:352-156429 
of bounded self-adjoint operators on a non-trivia $k$-Hilbert space making $s(A)$ a positive semidefinite operator for every $s \in S$. The quadratic module $M_{S}$ is the set of all elements of the form $\sum_{i} g_{i}^{*} s_{i} g_{i}$, where $s_{i} \in S \cup\{1\}$ and $g_{i} \in k\langle\bar{X}\rangle$, i.e., the smallest subset of $\operatorname{Sym} k\langle\bar{X}\rangle$ satisfying $\{1\} \cup S \subseteq M_{S}, M_{S}+M_{S} \subseteq M_{S}$ and $g^{*} M_{S} g \subseteq M_{S}$ for all $g \in k\langle\bar{X}\rangle$. We call $M_{S}$ archimedean if there exists $N \in \mathbb{N}$ with $N-\left(X_{1}^{2}+\cdots+X_{n}^{2}\right) \in M_{S}$. In this case, $K_{S}$ is bounded: There exists $N \in \mathbb{N}$ such that $N-\left(A_{1}^{2}+\cdots+A_{n}^{2}\right) \geq 0$ whenever $\left(A_{1}, \ldots, A_{n}\right) \in K_{S}$. In other words, the operator norm is bounded uniformly for all operators appearing in a tuple belonging to $K_{S}$. As in the commutative case [JP], boundedness of $K_{S}$ does not imply that $M_{S}$ is archimedean, see Example 1.1 below. Nevertheless, if $K_{S}$ is bounded, we can add $N-\left(X_{1}^{2}+\cdots+X_{n}^{2}\right)$ to $S$ for a sufficiently big $N \in \mathbb{N}$ to make $M_{S}$ archimedean without changing $K_{S}$.

1.1. Example. Let $n=2$ and write $\bar{X}=(X, Y)$, i.e., we consider $k\langle\bar{X}\rangle=k\langle X, Y\rangle$. For

$$
S:=\{X-1, Y-1,8-X Y-Y X\},
$$

$K_{S}$ is bounded, but $M_{S}$ is not archimedean.

To see the latter, consider the $k$-algebra homomorphism $\pi: k\langle X, Y\rangle \rightarrow k[X, Y]$ making the variables $X$ and $Y$ commute: Like in [JP. Example 4.6], one sees that there is no $N \in \mathbb{N}$ such that $N-\left(X^{2}+Y^{2}\right) \in \pi\left(M_{S}\right)$.

Unlike in the commutative case, we have to work a bit to show that $K_{S}$ is bounded: Let $(A, B) \in K_{S}$ and set $C:=A+B, D:=A-B$. Then $C+D \geq 2$, $C-D \geq 2$ and $C^{2}-D^{2} \leq 16$. Considering the operator norms $c:=\|C\|$ and $d:=\|D\|$, we get $d \leq c-2$ from $\pm D \leq C-2$ and $c^{2} \leq 16+d^{2}$ from the corresponding fact with capital letters. This implies $c^{2} \leq 16+(c-2)^{2}=16+c^{2}-4 c+4$, i.e., $c \leq 5$ and $d \leq 3$. We finally obtain $2\left\|A^{2}+B^{2}\right\|=\left\|C^{2}+D^{2}\right\| \leq c^{2}+d^{2} \leq 34$, whence $17-A^{2}-B^{2} \geq 0$.

In our terminology, the main result of $[\mathrm{HM}$ ] characterizes symmetric polynomials (everywhere) positive semidefinite on (bounded) $K_{S}$ with $M_{S}$ archimedean and can be stated as follows:

1.2. Theorem (Theorem 1.2 in $[\mathrm{HM}]$ ). Let $S \cup\{f\} \subseteq \operatorname{Sym} k\langle\bar{X}\rangle$ and suppose that $M_{S}$ is archimedean. If $f(A)>0$ for all $A \in K_{S}$, then $f \in M_{S}$.

Together with the following proposition whose proof is left to the reader, we see that under the assumptions of the preceding theorem, $f$ is positive semidefinite on $K_{S}$ if and only if $f+\varepsilon \in M_{S}$ for all $\varepsilon \in \mathbb{R}_{>0}$.

1.3. Proposition. Let $S \cup\{f\} \subseteq \operatorname{Sym} k\langle\bar{X}\rangle$. If $f \in M_{S}$, then $f(A) \geq 0$ for all $A \in K_{S}$.

Our main result characterizes symmetric polynomials that are nowhere negative semidefinite on $K_{S}$ with $M_{S}$ archimedean. We are indebted to Prof. Dr. J. Cimprič who motivated us to look at this problem. After having completed this work, we have received his work [C2] containing a different and abstract approach to the same problem (see [C2, Theorem 5]).

\footnotetext{
${ }^{1}$ In contrast to $[\mathrm{HM}]$, we exclude the trivial Hilbert space $\{0\}$. This choice does of course not affect the validity of Theorem 1.2 below $[\mathrm{HM}$, Theorem 1.2] but is necessary for Theorem 1.4 to hold.
} 
1.4. Theorem (Nirgendsnegativsemidefinitheitsstellensatz). The following conditions are equivalent for all $S \cup\{f\} \subseteq \operatorname{Sym} k\langle\bar{X}\rangle$ provided that $M_{S}$ is archimedean:

(i) $f(A) \not \leq 0$ for all $A \in K_{S}$.

(ii) There exist $r \in \mathbb{N}$ and $h_{1}, \ldots, h_{r} \in k\langle\bar{X}\rangle$ with $\sum_{i=1}^{r} h_{i}^{*} f h_{i} \in 1+M_{S}$.

As an application, we characterize polynomials that are positive semidefinite on the 'noncommutative cube' as those polynomials that can be approximated by sums of hermitian squares. Here, the points of the 'noncommutative cube' are tuples of contractions. A contraction is a bounded operator $A$ with operator norm $\|A\| \leq 1$. The approximation is with respect to the 1-norm which we define for any polynomial $f=\sum_{\alpha} a_{\alpha} \bar{X}^{\alpha}$ in commuting or noncommuting variables with coefficients $a_{\alpha}$ as

$$
\|f\|_{1}:=\sum_{\alpha}\left|a_{\alpha}\right|
$$

1.5. Theorem. Suppose $f \in k\langle\bar{X}\rangle$ is a polynomial of degree $d$, and set $s:=\sum_{i=0}^{d} n^{i}$. The following are equivalent:

(i) $f\left(A_{1}, \ldots, A_{n}\right)$ is positive semidefinite for all $k$-Hilbert spaces $E$ and all contractive self-adjoint operators $A_{1}, \ldots, A_{n}$ on $E$.

(ii) $f\left(A_{1}, \ldots, A_{n}\right)$ is positive semidefinite for all contractive self-adjoint matrices $A_{1}, \ldots, A_{n} \in k^{s \times s}$.

(iii) $f \in \overline{M_{\varnothing}}$ with respect to the 1 -norm, i.e., for all $\varepsilon \in \mathbb{R}_{>0}$, there exist $r \in \mathbb{N}$ and $g_{1}, \ldots, g_{r} \in k\langle\bar{X}\rangle$ such that

$$
\left\|f-\sum_{\ell=1}^{r} g_{\ell}^{*} g_{\ell}\right\|_{1}<\varepsilon .
$$

This result can be viewed as a noncommutative analog of the following well known result of Berg, Christensen and Ressel.

1.6. Theorem ( $\S 9$ in $[\overline{B C R}])$. For every polynomial $f \in \mathbb{R}\left[Y_{1}, \ldots, Y_{n}\right]$ in commuting variables $Y_{i}$, the following are equivalent:

(i) $f \geq 0$ on the cube $[-1,1]^{n}$.

(ii) For all $\varepsilon \in \mathbb{R}_{>0}$, there exist $r \in \mathbb{N}$ and $g_{1}, \ldots, g_{r} \in \mathbb{R}\left[Y_{1}, \ldots, Y_{n}\right]$ such that $\left\|f-\sum_{\ell=1}^{r} g_{\ell}^{*} g_{\ell}\right\|_{1}<\varepsilon$.

\section{Proofs}

2.1. Definition. To every quadratic module $M \subseteq k\langle\bar{X}\rangle$ we associate its ring of bounded elements

$$
H(M):=\left\{f \in k\langle\bar{X}\rangle \mid N-f^{*} f \in M \text { for some } N \in \mathbb{N}\right\} .
$$

This is indeed a ring, even a $k$-subalgebra of $k\langle\bar{X}\rangle$ as proved in [Vid, Lemma 4] (see also [C1, Section 2] and [S2, Section 2]).

2.2. Proposition. A quadratic module $M \subseteq k\langle\bar{X}\rangle$ is archimedean if and only if $H(M)=k\langle\bar{X}\rangle$.

Proof. Suppose $N \in \mathbb{N}$ is such that

$$
N-\left(X_{1}^{2}+\cdots+X_{n}^{2}\right) \in M
$$


It follows that

$$
N-X_{i}^{2}=N-\left(X_{1}^{2}+\cdots+X_{n}^{2}\right)+\sum_{j \neq i} X_{j}^{2} \in M,
$$

so $X_{i} \in H(M)$ for all $i$. Since $H(M)$ is a $k$-algebra, this implies that $H(M)=$ $k\langle\bar{X}\rangle$.

In the terminology of Köthe [Köt], this proposition together with the identity

$$
s=\left(\frac{s+1}{2}\right)^{2}-\left(\frac{s-1}{2}\right)^{2}
$$

shows that $M$ is an archimedean quadratic module if and only if 1 is an algebraic interior point of the convex cone $M \subseteq \operatorname{Sym} k\langle\bar{X}\rangle$. Recall: $f \in M$ is an algebraic interior point if for every $p \in \operatorname{Sym} k\langle\bar{X}\rangle$ there exists $\varepsilon>0$ with $f+\varepsilon p \in M$.

Proof of Theorem 1.4. The easy part is to show that (ii) implies (i) (for which the archimedean property of $M_{S}$ is not needed). Suppose $\sum_{i} h_{i}^{*} f h_{i} \in 1+M_{S}$ for some $h_{i} \in k\langle\bar{X}\rangle$. By Proposition 1.3 , $\left(h_{i}^{*} f h_{i}\right)(A) \geq 1$. For every non-zero vector $v \in E$,

$$
\sum_{i}\left\langle f(A) h_{i}(A) v, h_{i}(A) v\right\rangle=\sum_{i}\left\langle h_{i}^{*}(A) f(A) h_{i}(A) v, v\right\rangle \geq\langle v, v\rangle>0 .
$$

And hence (compare the footnote on page 2$) f(A) \not \leq 0$.

The proof of the non-trivial part proceeds in several steps.

STEP 1: Separation.

Let $C$ denote the set of all (finite) sums of elements $h^{*} f h(h \in k\langle\bar{X}\rangle)$. This is a convex cone in the $\mathbb{R}$-vector space $\operatorname{Sym} k\langle\bar{X}\rangle$. We claim that $C \cap\left(1+M_{S}\right) \neq \varnothing$. Assume otherwise. Then $C$ contains no algebraic interior points of $M_{S}$. Thus we can apply Eidelheit's separation theorem [Köt, §17.1(3)]. There exists a non-zero $\mathbb{R}$-linear functional $L_{0}: \operatorname{Sym} k\langle\bar{X}\rangle \rightarrow \mathbb{R}$ with $L_{0}\left(M_{S}\right) \subseteq \mathbb{R}_{\geq 0}$ and $L_{0}(C) \subseteq \mathbb{R}_{\leq 0}$. We now extend $L_{0}$ to a $k$-linear functional $L: k\langle\bar{X}\rangle \rightarrow k$ satisfying

$$
L\left(M_{S}\right) \subseteq \mathbb{R}_{\geq 0} \quad \text { and } \quad L(C) \subseteq \mathbb{R}_{\leq 0} .
$$

In case $k=\mathbb{R}$, we can choose $L$ as an arbitrary linear extension of $L_{0}$. Now consider the case $k=\mathbb{C}$. The identity

$$
p=\frac{p+p^{*}}{2}+i \frac{p-p^{*}}{2 i}, \quad p \in k\langle\bar{X}\rangle
$$

gives a direct sum decomposition of $\mathbb{C}\langle\bar{X}\rangle$ into two real vector spaces

$$
\mathbb{C}\langle\bar{X}\rangle=\operatorname{Sym} \mathbb{C}\langle\bar{X}\rangle \oplus i \operatorname{Sym} \mathbb{C}\langle\bar{X}\rangle .
$$

Now define $L$ by

$$
L(p+i q):=L_{0}(p)+i L_{0}(q), \quad p, q \in \operatorname{Sym} \mathbb{C}\langle\bar{X}\rangle .
$$

It is easy to check that $L$ is $\mathbb{C}$-linear.

STEP 2: The Gelfand-Naimark-Segal construction.

By the Cauchy-Schwarz inequality for semi-scalar products,

$$
N:=\left\{p \in k\langle\bar{X}\rangle \mid L\left(p^{*} p\right)=0\right\}
$$

is a linear subspace of $k\langle\bar{X}\rangle$. Similarly, we see that

$$
\langle\bar{p}, \bar{q}\rangle:=L\left(q^{*} p\right)
$$


defines a scalar product on $k\langle\bar{X}\rangle / N$, where $\bar{p}:=p+N$ denotes the residue class of $p \in k\langle\bar{X}\rangle$ modulo $N$. Let $E$ denote the completion of $k\langle\bar{X}\rangle / N$ with respect to this scalar product. Since $1 \notin N$ (otherwise $L=0$ because $M_{S}$ is archimedean), $E$ is non-trivial.

STEP 3: Construction of $\hat{X} \in K_{S}$.

To prove that $N$ is a left ideal of $k\langle\bar{X}\rangle$, we fix $i \in\{1, \ldots, n\}$ and show that $X_{i} N \subseteq$ $N$. Since $M_{S}$ is archimedean, there is some $m \in \mathbb{N}$ with $m-X_{i}^{2} \in M_{S}$ for every $i$. Hence for all $p \in k\langle\bar{X}\rangle$, we have

$$
0 \leq L\left(p^{*}\left(m-X_{i}^{2}\right) p\right) \leq m L\left(p^{*} p\right) .
$$

Now (3) shows that $L\left(p^{*} X_{i}^{2} p\right)=0$ for all $p \in N$, i.e., $X_{i} p \in N$.

Because $N$ is a left ideal, the map

$$
\Lambda_{i}: k\langle\bar{X}\rangle / N \rightarrow k\langle\bar{X}\rangle / N, \bar{p} \mapsto \overline{X_{i} p}
$$

is well-defined for each $i$. Obviously, it is linear and it is self-adjoint by the definition (2) of the scalar product. By (3), $\Lambda_{i}$ is bounded and thus extends to a self-adjoint operator $\hat{X}_{i}$ on $E$. We claim that $\hat{X}:=\left(\hat{X}_{1}, \ldots, \hat{X}_{n}\right) \in K_{S}$. For this, let $p \in S$ and $v \in E$ be arbitrary. Without loss of generality, $v=\bar{h}$ for some $h \in k\langle\bar{X}\rangle$. Hence

$$
\langle p(\hat{X}) v, v\rangle=\langle\overline{p h}, \bar{h}\rangle=L\left(h^{*} p h\right) \geq 0,
$$

because of $h^{*} p h \in M_{S}$ and (1).

Since $f$ is nowhere negative semidefinite on $K_{S}$, there is some $v \in E$ with $\langle f(\hat{X}) v, v\rangle>0$. As before, we may assume that $v=\bar{h}$ for some $h \in k\langle\bar{X}\rangle$. From (1), we get

a contradiction.

$$
0<\langle f(\hat{X}) \bar{h}, \bar{h}\rangle=\langle\overline{f h}, \bar{h}\rangle=L\left(h^{*} f h\right) \leq 0,
$$

We now turn to the proof of Theorem 1.5. The following proposition shows that all polynomial inequalities $f \geq 0$ holding for all self-adjoint (contraction) matrices are symmetric in the sense that $f=f^{*}$.

2.3. Proposition. If $f \in k\langle\bar{X}\rangle$ of degree $<d$ satisfies $f\left(A_{1}, \ldots, A_{n}\right)=0$ for all self-adjoint contractions $A_{1}, \ldots, A_{n} \in k^{d \times d}$, then $f=0$.

Proof. We form the $*$-polynomial $g:=f\left(\left(X_{1}+X_{1}^{*}\right) / 2, \ldots,\left(X_{n}+X_{n}^{*}\right) / 2\right)$ in noncommuting variables $X_{1}, \ldots, X_{n}, X_{1}^{*}, \ldots, X_{n}^{*}$. It follows from the assumption that $g\left(A_{1}, \ldots, A_{n}\right)=0$ for all contractions $A_{1}, \ldots, A_{n} \in k^{d \times d}$. Modify the multilinearization process explained in $\mathrm{Row}$ as follows: The formula for $\Delta_{i u}$ given in [Row, page 126] should be replaced by

$$
\begin{aligned}
\Delta_{i u} g= & g\left(X_{1}, \ldots, \frac{X_{i}+X_{u}}{2}, \ldots, X_{n}\right)-g\left(X_{1}, \ldots, \frac{X_{i}}{2}, \ldots, X_{n}\right) \\
& -g\left(X_{1}, \ldots, \frac{X_{u}}{2}, \ldots, X_{n}\right) .
\end{aligned}
$$

Repeating this, we obtain a multilinear $*$-polynomial of degree $<d$ killing all $d \times d$ contractions. But this is impossible by the general theory of polynomial identities (cf. Row, Remark 2.5.14]).

The following lemma implies Theorem 1.5 in the very special case $f=1-X_{i}^{2}$, which could also be deduced from Theorem 1.6. 
2.4. Lemma. For all $m \in \mathbb{N}, 1-Y^{2}+\frac{1}{m} Y^{2 m}$ is a sum of squares in the polynomial $\operatorname{ring} \mathbb{R}[Y]$.

Proof. Check that

$$
1-Y^{2}+\frac{1}{m} Y^{2 m}=\frac{1}{m}+\frac{1}{m}\left(1-Y^{2}\right)^{2} \sum_{k=0}^{m-2}(m-1-k) Y^{2 k} .
$$

Proof of Theorem 1.5. To see the easy implication from (iii) to (iii), let $A:=\left(A_{1}, \ldots, A_{n}\right)$ be a tuple of contractive self-adjoint $s \times s$ matrices. It is easy to see that (iii) implies $f=f^{*}$, in particular $f(A)=f(A)^{*}$. Fix a vector $v \in k^{s}$ with $\|v\|=1$. We show that

$$
\langle f(A) v, v\rangle>-\varepsilon
$$

for all $\varepsilon \in \mathbb{R}_{>0}$. Choose $r \in \mathbb{N}$ and $g_{1}, \ldots, g_{r} \in k\langle\bar{X}\rangle$ such that $\|h\|_{1}<\varepsilon$ for $h:=f-\sum_{\ell=1}^{r} g_{\ell}^{*} g_{\ell}$. Note that $h(A)$ is self-adjoint whence

$$
\langle f(A) v, v\rangle=\langle h(A) v, v\rangle+\sum_{\ell=1}^{r}\left\langle g_{\ell}(A) v, g_{\ell}(A) v\right\rangle \geq-\|h(A)\| \geq-\|h\|_{1}>-\varepsilon .
$$

Let us now prove that (ii) implies (ii) by contraposition. We assume that there exists a tuple $A:=\left(A_{1}, \ldots, A_{n}\right)$ of self-adjoint contractions on a $k$-Hilbert space $E$ for which $f(A)$ is not positive semidefinite. Let $v \in E$ be a vector with $\langle f(A) v, v\rangle<$ 0 and define

$$
V:=\operatorname{Span}\left\{A^{w} v \mid w \text { is a word of length } \leq d\right\} .
$$

$V$ is a finite dimensional $k$-vector space with $\operatorname{dim} V \leq s$. Let $\pi: E \rightarrow V$ denote the orthogonal projection and define $B_{i}:=\pi A_{i} \pi$. Obviously, $B_{i}$ is a self-adjoint contraction and

$$
\left\langle f\left(B_{1}, \ldots, B_{n}\right) v, v\right\rangle=\langle f(A) v, v\rangle<0 .
$$

It remains to show that (i) implies (iii). Assume that (ii) holds. Since $f+\frac{1}{m}$ converges to $f$ in the 1 -norm when $m \rightarrow \infty$, we may assume that $f$ is positive definite for all $n$-tuples of self-adjoint contractive operators. Let $S:=\left\{1-X_{1}^{2}, \ldots, 1-X_{n}^{2}\right\}$. By assumption, $f$ is positive definite on $K_{S}$ and $M_{S}$ is archimedean. Thus by Theorem 1.2, $f \in M_{S}$, i.e., $f$ can be written as

$$
f=\sum_{i} g_{i}^{*} g_{i}+\sum_{j=1}^{n} \sum_{i} g_{i j}^{*}\left(1-X_{j}^{2}\right) g_{i j}
$$

for some $g_{i}, g_{i j} \in k\langle\bar{X}\rangle$. Hence it suffices to show that $g^{*}\left(1-X^{2}\right) g \in \overline{M_{\varnothing}}$ for every $g \in k\langle\bar{X}\rangle$. Identifying $\mathbb{R}[Y]$ from Lemma 2.4 with $\mathbb{R}\left[X_{j}\right] \subseteq k\langle\bar{X}\rangle$, we see $1-X_{j}^{2} \in$ $\overline{M_{\varnothing}}$. Noting that the map $k\langle\bar{X}\rangle \rightarrow k\langle\bar{X}\rangle, p \mapsto g^{*} p g$ is bounded with respect to the 1-norm (left or right multiplication by any variable is even an isometry), finishes the proof.

\section{Concluding Remarks}

In Theorem 1.4 the hypothesis that $M_{S}$ is archimedean cannot be dropped. Otherwise boundedness of $K_{S}$ would imply that $M_{S}$ is archimedean (contradicting Example 1.1). In the commutative case, the analogous implication holds often (see [Mar, JP]), for example when $S$ is a singleton (a quadratic module generated by one element is a preordering and for preorderings Schmüdgen's Theorem S2, 
Corollary 3] holds). In the noncommutative case, the situation is different: In the next example, $S$ is a singleton, $K_{S}=\varnothing$ and yet $M_{S}$ is not archimedean.

3.1. Example. Let $n=2, k=\mathbb{C}$ and write $\bar{X}=(X, Y)$, i.e., we consider $k\langle\bar{X}\rangle=$ $\mathbb{C}\langle X, Y\rangle$. Set

$$
S:=\left\{-(X Y-Y X+i)(X Y-Y X+i)^{*}\right\} .
$$

We claim that $K_{S}=\varnothing$ but $M_{S}$ is not archimedean (though $S$ is a singleton).

Assume that there exists $(A, B) \in K_{S}$. Then $A B-B A=-i$ and by induction $A^{m} B-B A^{m}=-i m A^{m-1}$ for all $m \in \mathbb{N}$. Since $A$ is self-adjoint, $\left\|A^{m}\right\|=\|A\|^{m}$ and therefore

$$
m\|A\|^{m-1}=\left\|A^{m} B-B A^{m}\right\| \leq 2\|A\|^{m}\|B\|
$$

for all $m \in \mathbb{N}$. Noting that $A \neq 0$, this yields that $m \leq 2\|A\|\|B\|$ for all $m \in \mathbb{N}$, a contradiction. Therefore $K_{S}=\varnothing$.

In order to show that $M_{S}$ is not archimedean, we use the Schrödinger representation (cf. [S2])

$$
\begin{aligned}
\pi_{0}: \mathbb{C}\langle X, Y\rangle & \rightarrow \mathcal{L}(S(\mathbb{R})) \\
X & \mapsto\left(f \mapsto-i f^{\prime}\right) \\
Y & \mapsto(f \mapsto(x \mapsto x f(x)))
\end{aligned}
$$

where $S(\mathbb{R})$ is the dense subspace of $L^{2}(\mathbb{R})$ consisting of all Schwartz (i.e., 'rapidly decreasing smooth') functions $\mathbb{R} \rightarrow \mathbb{C}, \mathcal{L}(S(\mathbb{R}))$ is the vector space of all $\mathbb{C}$-linear operators $S(\mathbb{R}) \rightarrow S(\mathbb{R})$ and $f^{\prime}$ denotes the derivative of $f \in S(\mathbb{R})$. Note that $\pi_{0}$ is a $\mathbb{C}$-algebra homomorphism which respects the involution $*$ since $\pi_{0}(X), \pi_{0}(Y)$ are self-adjoint (for $\pi_{0}(X)$ this follows from integration by parts). Observing that $\pi_{0}(X Y-Y X+i)=0$, we get that $\pi_{0}$ sends every element of $M_{S}$ to a positive semidefinite operator on $S(\mathbb{R})$. Finally, $N-\pi_{0}\left(Y^{2}\right) \nsupseteq 0$ regardless of $N \in \mathbb{N}$.

In spite of the last example, we do not know if the Nirgendsnegativsemidefinitheitsstellensatz 1.4 holds for $S=\varnothing$ (where $M_{S}$ fails to be archimedean).

3.2. Open Problem. Regard the following conditions for a symmetric polynomial $f \in k\langle\bar{X}\rangle$.

(i) $f\left(A_{1}, \ldots, A_{n}\right)$ is not negative semidefinite for any $k$-Hilbert space $E \neq\{0\}$ and bounded self-adjoint operators $A_{1}, \ldots, A_{n}$ on $E$.

(ii) There are $r \in \mathbb{N}$ and $g_{1}, \ldots, g_{r} \in k\langle\bar{X}\rangle$ such that $\sum_{i=1}^{r} g_{i}^{*} f g_{i} \in 1+M_{\varnothing}$.

Obviously, (ii) implies (ii). Does (i) imply (ii)?

An affirmative answer to this problem would give a noncommutative analog of Artin's solution to Hilbert's 17th problem (see for example [Rez or [Mar]).

Acknowledgments. We thank Prof. Dr. K. Schmüdgen for bringing Eidelheit's separation theorem to our attention. Our sincere thanks also go to Prof. Dr. J. Cimprič whose talk at the Luminy conference on Positive Polynomials brought up the idea of investigating nowhere negative semidefinite polynomials.

\section{REFERENCES}

[BCR] C. Berg, J. Christensen, P. Ressel: Positive definite functions on Abelian semigroups, Math. Ann. 223, 253-274 (1976)

[C1] J. Cimprič: A representation theorem for archimedean quadratic modules on *-rings, Canad. Math. Bull. (to appear) 
[C2] J. Cimprič: Maximal quadratic modules on *-rings, Algebr. Represent. Theory (to appear)

[Hel] J. W. Helton: "Positive" noncommutative polynomials are sums of squares, Ann. Math. (2) 156, No. 2, 675-694 (2002)

[HM] J. W. Helton, S. McCullough: A Positivstellensatz for non-commutative polynomials, Trans. Amer. Math. Soc. 356, 3721-3737 (2004)

[JP] T. Jacobi, A. Prestel: Distinguished representations of strictly positive polynomials, J. Reine Angew. Math. 532, 223-235 (2001)

[Köt] G. Köthe: Topological vector spaces I, Springer-Verlag, Berlin, 1969

[Mar] M. Marshall: Positive polynomials and sums of squares, Istituti Edit. Poligraf. Int., Pisa, Roma (2000)

[MP] S. McCullough, M. Putinar: Non-commutative sums of squares, Pacific J. Math. 218, 167-171 (2005)

[Rez] B. Reznick: Some concrete aspects of Hilbert's 17th problem, Contemp. Math. 253, 251$272(2000)$

[Row] L. Rowen: Polynomial identities in ring theory, Pure and Applied Mathematics, Vol. 84. New York, Academic Press (1980)

[S1] K. Schmüdgen: The $K$-moment problem for compact semi-algebraic sets, Math. Ann. 289, No. 2, 203-206 (1991)

[S2] K. Schmüdgen: A strict Positivstellensatz for the Weyl algebra, Math. Ann. 331, No. 4, 779-794 (2005)

[Vid] I. Vidav: On some *-regular rings, Acad. Serbe Sci., Publ. Inst. Math. 13, 73-80 (1959)

Igor Klep, Univerza v Ljubljani, Oddelek za matematiko InŠtituta za matematiko, Fiziko in mehaniko, Jadranska 19, 1111 Ljubljana, Slovénie

E-mail address: igor.klep@fmf.uni-lj.si

Markus Schweighofer, Universität Konstanz, Fachbereich Mathematik und Statistik, 78457 Konstanz, Allemagne

E-mail address: markus.schweighofer@uni-konstanz.de 